\title{
Long and short Integrated Management of Childhood Illness (IMCI) training courses in Afghanistan: a cross- sectional cohort comparison of post-course knowledge and performance
}

\author{
Maureen Mayhew ${ }^{1 *}$, Paul Ickx² , William Newbrander ${ }^{2}$, Hedayatullah Stanekzai $^{3}$, Sayed Alisha Alawi ${ }^{4}$
}

\begin{abstract}
Background: In 2003 the Afghan Ministry of Public Health (MoPH) adopted the Integrated Management of Childhood Illness (IMCI) for delivering child health services in primary care facilities. Key problems were subsequently identified: high cost of training, frequent health worker turnover and poor quality of IMCI implementation by those trained - specifically in the use of job aids and protocols for assessment, classification, treatment and counselling. The high financial, human resources and opportunity costs of implementing IMCI spurred the MoPH to prioritize developing a shortened IMCI course of comparable quality to the 11-Day training.

Methods: This cross-sectional evaluation compared knowledge before and after training, and health worker performance in assessment, classification and treatment of sick children in two similar cohorts, eight months post-training.

Results: The mean increase in knowledge scores of the thirty 7-Day course trainees was 29 [95\% Confidence Interval (CI): 24, 34] compared to 23 (95\% CI: 18, 28) in the 31 trained in the 11-Day course. During assessment visits, mean scores in the 7-Day course trainees and the 11-Day course trainees were $93 \%$ (95\% CI: 91, 95) versus $94 \%$ (95\% CI: 91, 96) in assessment; 95\% (95\% CI: 89, 100) versus $96 \%(95 \%$ CI: 91,100$)$ in classification; $95 \%$ (95\% CI: 92, 100) versus $97 \%$ ( $95 \%$ CI: 95,100$)$ in treatment; and $81 \%(95 \%$ CI: 76,86$)$ versus $80 \%(95 \%$ CI: 75,85$)$ in counselling. The 7 -Day course was $36 \%$ less expensive than the 11-Day course. For each course opportunity costs, measured as numbers of children who potentially received poorer care than usual during trainee absence, were 3,160 for the 11-Day course and 2,016 for the 7-Day course. This measure was chosen because trainee absence commonly resulted in higher patient volumes per remaining provider or complete closure of a health facility with one single health worker.

Conclusion: Given similar performance and knowledge of health workers trained in both courses, potential cost savings, the possibility of training more health workers and the relative ease with which health workers in remote settings might participate in a shorter course, it seems prudent to standardize the 7-Day course in Afghanistan where child mortality rates remain unacceptably high.

Keywords: Child Health, Integrated Management of Childhood Illness (IMCI), In-Service, Training, Afghanistan Copyright: $\odot 2015$ by Kerman University of Medical Sciences

Citation: Mayhew M, Ickx P, Newbrander W, Stanekzai H, Alawi SA. Long and short Integrated Management of Childhood Illness (IMCI) training courses in Afghanistan: a cross-sectional cohort comparison of post-course knowledge and performance. Int J Health Policy Manag 2015; 4: 143-152. doi: 10.15171/ijhpm.2015.17
\end{abstract}

\section{Article History:}

Received: 6 october 2014 Accepted: 23 January 2015 ePublished: 27 January 2015

\section{*Correspondence to:}

Maureen Mayhew

Email: maureen.mayhew@ubc.ca

\section{Key Messages}

Implications for policy makers

- The performance of health worker trainees in the 7-day and 11-day Integrated Management of Childhood Illness (IMCI) training courses were similar at a fourth monitoring visit, 32 weeks after training

- The 7-Day course was $36 \%$ less expensive than the 11-Day course.

- The 7-day training course offers the possibility of training more health workers and allows health workers in remote settings a greater opportunity to participate in an IMCI training course

- By training more health workers from different disciplines, fewer opportunity costs related to missed vaccines, untreated health concerns and inappropriate treatment or follow-up are expected.

Implications for public

When compared to the standard course, the shorter course ensures equally knowledgeable clinicians who can provide more continuous care at health facilities, because the health workers are absent for a shorter period of time during the course, an especially important concept in remote areas with few or no alternatives. More health workers can be trained in the same period of time, resulting in a larger pool of health workers, and more likelihood that children will be attended by health workers trained in Integrated Management of Childhood Illness (IMCI). Nurses and midwives, who see many children in Afghanistan, provide good quality care after being trained in the short IMCI course. 


\section{Introduction}

In 2002, the Ministry of Public Health (MoPH) of the new government of the Islamic Republic of Afghanistan faced major child health challenges that resulted in high mortality in children less than five years of age. One in four children in this age group died from preventable or curable diseases, such as pneumonia, diarrhoea, measles, malaria and malnutrition $(1,2)$. The MoPH decided to adopt, as an integral part of the Basic Package of Health Services (BPHS), the Integrated Management of Childhood Illness (IMCI), a strategy developed in 1992 by the United Nations Children's Fund (UNICEF) and the World Health Organization (WHO) to reduce mortality and morbidity in children under five years of age $(3,4)$. IMCI encourages the use of proven interventions for curative and preventative care to deal with the major illnesses that, in Afghanistan, commonly affect children less than five years old. These interventions include immunization, oral rehydration therapy, antibiotics, and appropriate infant nutrition.

IMCI has been widely adopted by many developing countries and has been shown to be of more benefit in low-income countries (5-7). Several challenges have impeded the scale up of IMCI including insufficient numbers of trained health workers, high cost of training and the sad reality that during the absence of health workers for training, most sick children received no treatment $(5,8)$. Studies have highlighted several influential factors on health worker performance of IMCI in addition to course characteristics. These include health system factors (e.g. the presence of essential medicines and equipment, adequate supervision after training), health worker factors (e.g. gender and job satisfaction), and community or household factors that impact quality care provided to the child in the household (9-12). In 2007, WHO recommended that alternative courses to the standard training should be investigated, but the absence of standard guidance tools on how best to shorten the 11-Day course have resulted in several countries designing wide-ranging courses of five to ten days in duration, some of which contain no practical exercises in the classroom part of the course and little practice time in health facilities $(6,13-15)$.

Thus far, a key challenge to developing shorter IMCI courses has been the apprehension of reduced quality of care provided by those trained for a shorter period (15). In response to this concern, Rowe et al. conducted a systematic review to identify IMCI effectiveness in the standard 11-Day IMCI training compared to shorter courses. They concluded: "Based on limited evidence, standard training (11-Days) for health workers on IMCI guidelines for managing ill children seemed more effective than short training (5 to 10-Days), although the differences might be small". That said, the authors noted that "there were too few direct comparisons of standard and short training with first-tier study designs to conclude firmly whether shortening IMCI training reduces its effectiveness (and if so, to what degree effectiveness is reduced)". They and others pointed out that reduced training costs, savings ranging from $13 \%$ to $46 \%$, were associated with all of the shortened IMCI courses $(13,16)$.

In 2008 the Afghan MoPH's IMCI working group, with support from Basic Support for Institutionalizing Child Survival (BASICS), UNICEF and WHO, reviewed the IMCI program and found that in spite of having trained 2,134 health workers in IMCI, sick children were not properly assessed and treated by trainees, and that their parents were poorly advised (17). The primary problems identified by the working group were similar to issues identified in other countries: insufficient numbers of health workers were trained in IMCI and there was high attrition amongst those trained $(8,18)$. Previous assessments of the quality of care provided in the Afghan primary health system have highlighted several additional influences on quality of IMCI implementation: availability of doctors, having ever received standard IMCI training, short appointment times for patients due to large patient volumes, and factors such as provider job satisfaction, availability of clinical guidelines, frequency of supervision and the presence of community councils $(9,10,19,20)$. In Afghanistan $71 \%$ of IMCI trainees were physicians when the more common types of health workers who provided services to sick children were midwives, nurses and community health workers (17). Fifty percent of primary care facilities had no IMCI trained health worker. Minimal supervision was provided to health workers after training and consequently, the quality of the assessment and treatment of sick children was poor. Other concerns included the high cost of the 11-Day IMCI training and the length of time trainees remained away from health facilities during which time care provided to children was of a lesser quality than usual. After careful consideration of the preceding issues, the MoPH prioritized developing a shortened and updated IMCI course, and requested an evaluation of the shortened course prior to scale-up.

The 11-Day course for Afghanistan had been developed in 2003 and consisted of seven modules and several job aids including a Chart Booklet that contained Afghan-specific counselling information, examples and exercises. A review of the shortened courses previously developed in Pakistan, Benin, Uganda, India, Nigeria, Kosovo, and Zambia revealed that in most of these, shortening the courses resulted in reducing time spent on practical application of the basic knowledge acquired from the course, specifically time spent doing exercises and practice sessions in clinics and hospitals. In Afghanistan, the standard 11-Day course was shortened by condensing the general science information while maintaining practice in applying knowledge through exercises and on-site clinic or hospital sessions, and by replacing the inclass readings by brief discussions of the highlights of each topic with assigned readings at home. The shortened course was streamlined into five modules by combining module 1 (Introduction) and module 2 (Assess and Classify Sick Child), and combining module 3 (Identify Treatment) and module 4 (Treat the Child). The ensuing modules focused on how to classify and prioritize illnesses, how to treat classifications, how to counsel, important assessment and treatment in younger children, and when to follow-up. To promote active adult-focused, participatory learning, nearly all of the exercises and clinical training were retained in the shortened curriculum. Strong emphasis on using the IMCI Chart Booklet (a job aid for the health worker) throughout the entire course was intended to promote regular, routine use of the tool by the health workers when seeing sick children. Using information from WHO's 2008 version of IMCI, the Chart Booklet and recording forms were revised and updated 
to include new treatments as well as the new classifications of otitis media, sore throat and jaundice in the young child. Some diagrams were simplified and culturally appropriate images and nutritional information were provided.

The 7-Day curriculum was pilot tested on a group of five NonGovernment Organization (NGO)-employed physicians and three $\mathrm{MoPH}$ child health staff, all of whom had previously received the standard 11-Day IMCI training. Before launching the 7-Day curriculum, feedback from these participants was used to modify the curriculum, the recording forms and the Chart Booklet. All training was conducted at the Afghan MoPH IMCI department by MoPH IMCI staff and BASICS technical officers in Kabul. From January to April 2010 two 11-Day courses and three 7-Day courses were conducted. Because the short course was updated to include additional clinical information not contained in the 11-Day Afghan course, the evaluation was directed only at topics common to both.

The purpose of this evaluation was to answer questions regarding quality of the 7-Day training in physicians and non-physicians by undertaking a comparison of the level of knowledge and performance in assessment and treatment of sick children by health workers who were trained in the 7-Day course compared to those trained in the standard 11-Day course. This manuscript illustrates a process of adapting a global standard approach to local needs, and using implementation research to address concerns not answered by available evidence.

\section{Methods}

This cross-sectional program evaluation compared knowledge gained and converted into practice in two similar cohorts of practicing healthcare workers each trained in one of two inservice training courses - the standard 11-Day IMCI course or a 7-Day IMCI course that was developed from WHO's standard 11-Day course. The two purposively selected groups of health workers from eight districts of Takhar province were matched as much as possible for gender, health worker type (doctor, nurse or midwife), proximity to a regional centre, type of health facility where the health worker was stationed and previous IMCI course participation. Health workers from primary healthcare facilities-Basic Health Centres (BHC) and Comprehensive Health Centres (CHC)-were selected by NGO administrators. Preference was given to those who had not previously taken an IMCI course but, according to NGO supervisors, five health workers who had previously taken the course were in need of additional training. Due to ethical considerations regarding quality of care, rather than exclude this group, they were admitted into the course where it was identified that their pre-test knowledge was no better than those who had not previously taken the course and their knowledge gains were similar to other trainees. Knowledge pre- and post-training were compared between the two cohorts and a direct comparison of IMCI practice outcomes, characterized by adherence to IMCI protocols, was conducted through directly observing trainees at health centres 30 to 32 weeks post-training during a fourth observation visit. Due to resource constraints the sample size was based on detecting a $10 \%$ difference in knowledge scores between two matched samples.
Thirty-one participants of the 7-Day course were selected from four districts of Takhar Province (Chal, Chah Ab, Khwaja Bahaudin and Farkhar) and completed the training. One physician in the 7-Day course did not do the pre-test so he was excluded from the post-test knowledge score calculation but he was observed during the assessment visits. Thirtyone participants for the 11-Day course were selected from three districts of Takhar (Rustaq, Khwaja Ghar and Warsaj). Midwives, nurses and physicians were trained together. Because of funding, distance, time and logistical constraints, the five physicians chosen by the NGO administrators who had previously taken the course were distributed between the two groups rather than omitting them from the analysis. In Table 1 attrition due to staff turnover in both groups is portrayed.

An estimate of costs of both training courses was determined by listing fixed and variable cost inputs. Any cost that was classified as fixed was not included in the analysis since it was assumed the same for both courses.

The MoPH's intent in leading this program was to improve the quality of existing, routine health service delivery to children by adapting, updating and shortening an existing IMCI curriculum to which the Afghan government had experienced major implementation challenges. With the goal of improving care and expanding IMCI training, $\mathrm{MoPH}$ requested this program evaluation to identify any change in health worker knowledge and the resultant provision of care. Standard MoPH procedures for consent and anonymity were followed in this evaluation. The consent of health workers was implied when they accepted the offer of registering for an IMCI course following which supervisory visits, that were focused on reinforcing clinical knowledge and skills acquired from the course, were routinely provided by $\mathrm{MoPH}$ staff or delegates and from which data were collected. Patients sought and received care in the usual fashion from healthcare providers. No one was refused care. No patient or health worker identifiers were collected and only aggregate data on classification and treatment was used for analysis of health worker performance.

\section{Data collection \\ Pre-test and post-test knowledge}

Questions for pre- and post-tests pertained only to knowledge regarding children aged 2-59 months and to those classifications and treatments that were common to both courses (Additional file 1). The post-test was identical to the pre-test administered at the end of each course prior to discussing answers to any of the questions. Individual trainees recorded answers directly onto paper tests which were input into a database by the trainers.

\section{Direct observation}

The Sick Child Assessment and Treatment Observation Checklist for Validators was a modified and updated version of the standard IMCI observation checklist (Additional file 2). Assessors were physicians previously trained in the health worker IMCI clinical course and the IMCI Training of Trainers course, and had experience in routinely using IMCI algorithms for clinical practice as well as performing followup, on-site visits to IMCI trainees. They did not function as 
Table 1. Health workers' characteristics by course and fourth monitoring visit

\begin{tabular}{|c|c|c|c|c|}
\hline \multirow{2}{*}{$\mathrm{IMCl}$ course length } & \multicolumn{2}{|c|}{ Trained at baseline } & \multicolumn{2}{|c|}{$4^{\text {th }}$ monitoring visit } \\
\hline & 7-Day & 11-Day & 7-Day & 11-Day \\
\hline Planned visit & - & - & 32 & 32 \\
\hline Actual visit & 0 & 0 & $31.8(31.3,32.4)$ & $31.3(30.4,32.4)$ \\
\hline Health worker type & $\mathrm{N}(\%)^{*}$ & N (\%) & $N(\%)$ & N (\%) \\
\hline \multicolumn{5}{|l|}{ Medical doctor } \\
\hline Male & $6(100.0)$ & $9(90.0)$ & $5(100.0)$ & $7(100.0)$ \\
\hline Female & $0(0.0)$ & $1(10.0)$ & $0(0.0)$ & $0(0.0)$ \\
\hline Total & $6(19.4)^{+}$ & $10(32.2)^{+}$ & $5(20.0)^{+}$ & $7(29.2)^{+}$ \\
\hline \multicolumn{5}{|l|}{ Midwife } \\
\hline Male & $0(0.0)$ & $0(0.0)$ & $0(0.0)$ & $0(0.0)$ \\
\hline Female & $14(100.0)$ & $12(100.0)$ & $11(100.0)$ & $9(100.0)$ \\
\hline Total & $14(45.2)^{+}$ & $12(38.7)^{\dagger}$ & $11(44.0)^{+}$ & $9(37.5)^{+}$ \\
\hline \multicolumn{5}{|l|}{ Nurse } \\
\hline Male & $9(81.8)$ & $7(77.8)$ & $7(77.8)$ & $6(75.0)$ \\
\hline Female & $2(18.2)$ & $2(22.2)$ & $2(22.2)$ & $2(25.0)$ \\
\hline Total & $11(35.5)^{+}$ & $9(29.0)^{+}$ & $9(36.0)^{+}$ & $8(33.3)^{+}$ \\
\hline \multicolumn{5}{|l|}{ Gender of trainee } \\
\hline Male & $15(48.4)$ & $16(51.6)$ & $12(48.0)$ & $13(54.2)$ \\
\hline Female & $16(51.6)$ & $15(48.4)$ & $13(52.0)$ & $11(45.8)$ \\
\hline Health workers with previous IMCI training & $2(6.5)$ & $3(9.7)$ & $-\neq$ & $-\ddagger$ \\
\hline \multicolumn{5}{|l|}{ Type of health centre where trainee worked } \\
\hline Basic Health Center & $15(48.4)$ & $13(41.9)$ & $11(44.0)$ & $11(45.8)$ \\
\hline Comprehensive Health Center & $16(51.6)$ & $18(58.1)$ & $14(56.0)$ & $13(54.2)$ \\
\hline Total & $31(100)$ & $31(100)$ & $25(80.6)^{\# \#}$ & $24(77.4)^{\# \#}$ \\
\hline
\end{tabular}

$\mathrm{IMCl}=$ Integrated Management of Childhood Illness

*Percentages may not add to one hundred due to rounding.

${ }^{\dagger}$ Percentages of health worker type in each course group at baseline or at $4^{\text {th }}$ monitoring visit

‡Lost to follow-up due to attrition

\#\#Percentage of those trained at baseline who were observed during the $4^{\text {th }}$ monitoring visit.

the initial trainers or routine supervisors of the health workers in this evaluation. To ensure that their knowledge was upto-date, they completed an IMCI refresher course, and were subsequently trained in applying the observation checklist as assessors and validators, both for the 11-Day and the 7-Day algorithm. This training included role playing as assessor and as validator in the classroom followed by supervising practice visits in health centres. One assessor and one validator worked in pairs - the validator identified the correct classification and management, while the assessor completed the observation checklist. Each trainee was observed during four separate assessment visits planned at 6,12, 24 and 32-week intervals post-training. During each visit, the assessor and validator observed the health worker during three encounters with children 2 months to 5 years of age, calculated scores and then corrected any of the trainee's mistakes. For each sick child seen by the trainees, appropriateness of assessment, accuracy of classification and adherence to IMCI treatment guidelines were determined by comparing the observed checklist with the validator's assessment, classification, and treatment. The initial design of using the data from these visits as a time series was abandoned due to the high variability in elapsed time between completion of IMCI training and the observation visit. Observation checklists were verified twice for completeness - once by the assessor before leaving the health facility and once in Kabul by the IMCI trainers.

\section{Data analysis}

Data from all four assessment visits were put into an EXCEL database. Due to high variability in the timing of the initial three visits, data from the last visit only was retained for evaluation. Analysis was done using STATA version 11 (StataCorp LP, College Station, TX). Statistical significance of proportions was tested with Analysis of Variance (ANOVA) across health worker types and Chi-Square test statistic for between course comparisons. Means were tested using the T-Test statistic. Statistical significance for all outcome comparisons was set at 0.05 .

Pre-test and post-test knowledge

The outcome variables for knowledge gained during the course were calculated as the mean difference in post-test score compared to pre-test score per health worker then compared by health worker type and by course.

Direct observation of IMCI protocol adherence:

Five outcome scores of interest were compared during the observation visit.

1. Assessment tasks completed;

2. Correct classification;

3. Correct treatment for pneumonia, diarrhoea and dysentery;

4. Proportion of children requiring antibiotics and oral rehydration salts (ORS) who received them;

5. Counselling tasks completed.

The assessment score compared the mean proportion of completed assessment tasks for the 7-Day and 11-Day training groups. This score included variables that described the following tasks that had been observed by the assessors: all danger signs were verified; all main IMCI symptom questions were asked; and the proportion of the assessment tasks for cough, diarrhoea, fever and nutrition that had been 
completed during the assessment of the child.

The classification score compared the mean proportion of whether, for each child, the classification given by the health worker was in agreement with the classification assigned by the validator.

The treatment score compared the mean proportions of whether the children classified as pneumonia, diarrhoea or dysentery were correctly treated and whether treatments matched the treatment recommended by the validator.

The counselling score compared the mean proportion of IMCI counselling tasks performed by the health worker including mentioning to the caretaker three reasons to bring the child back to the health centre for follow-up.

Each of the scores for the two course groups were compared using bivariate analysis stratified by observation round and by health worker type. In order to fully explore potential sampling biases, outcome variables were analysed by health facility type and after excluding the five physicians who had previously taken an IMCI course.

\section{Cost estimates}

Included in the cost estimates of the trainings were the salaries of the trainers, travel costs for trainees, accommodation and meal costs for the trainees, and the cost of training materials. No training room rental costs were included since all courses were conducted in existing training or health facilities. Since the costs of travel and training materials were similar for the 7-Day and 11-Day course, these were considered identical and omitted from the analysis. Opportunity costs were estimated from the mean daily number of children less than five years of age who visited each primary care facility. These data were acquired from the Health Management Information System (HMIS), the surveillance data system of the MoPH that provided information on numbers, ages and diagnoses of patients visiting each BPHS facility. This measure was used since it is common in health facilities to have higher patient volumes per remaining provider during the absence of the trainee or the complete closure of the health facility in rural areas where there is only one health worker.

\section{Results}

More physicians were trained in the 11-Day course $(32.2 \%)$ than in the 7-Day course (19.4\%) and a higher proportion of nurses and midwives were trained in the 7-Day course $(80.6 \%)$ than in the 11-Day course (67.8\%). Gender distribution across trainee groups was similar: $51.6 \%$ female in the 7 -Day course and $48.4 \%$ female in the 11-Day course. All physicians included in the groups were male with the exception of one female physician in the 11-Day group. Nurses were more commonly male than female: $81.8 \%$ in the 7 -Day group and $77.8 \%$ in the 11 -Day group. All midwives were female. Similar proportions of health workers in Basic Health Centres (BHCs), the most remote type of health centre, occurred in each group. Three physicians had previously been trained in the 11-Day course and two had been previously trained in the 7-Day group. Health worker turn-over was high in both groups. By the fourth round, seven health workers from the 11-Day trainee group and six health workers from the 7-Day trainee group had resigned. The relative proportion of nurses in the 11-Day group rose from $29.0 \%$ to $33.3 \%$ over the period of observation. The proportions of health worker types remained the same for the 7-Day group. Table 1 illustrates the numbers and characteristics of trainees in the two courses at the end of the training course and at the conclusion of the fourth observation visit.

\section{Pre-test and post-test knowledge}

The simple boxplots demonstrate the distribution in pretest and post-test scores between the two course groups for each health worker category (Figures 1 and 2) and for posttest scores in combined health worker categories (Figure 3).

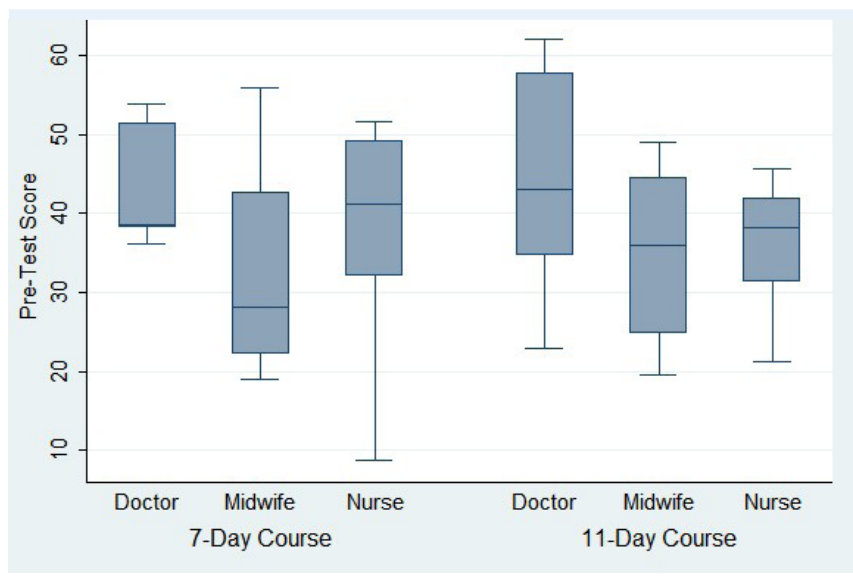

Figure 1. Pre-test score by health worker category and course

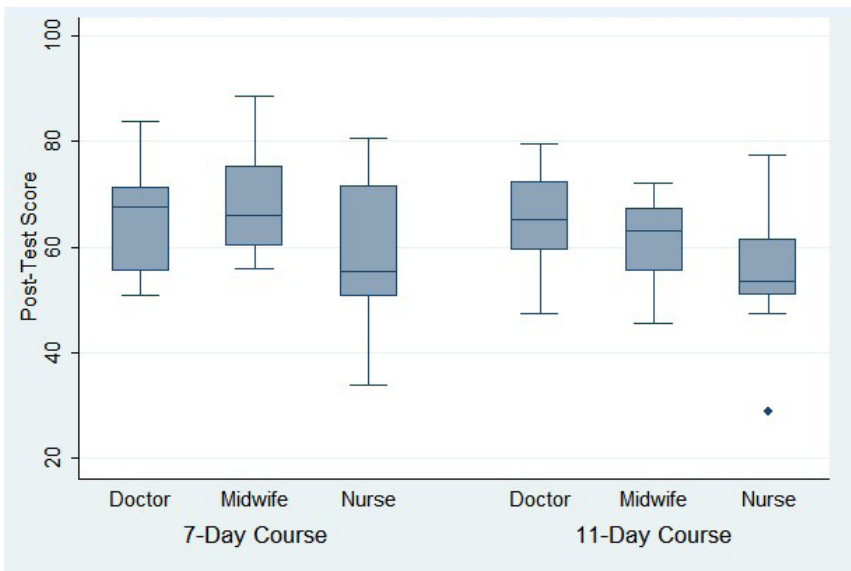

Figure 2. Post-test score by health worker category and course

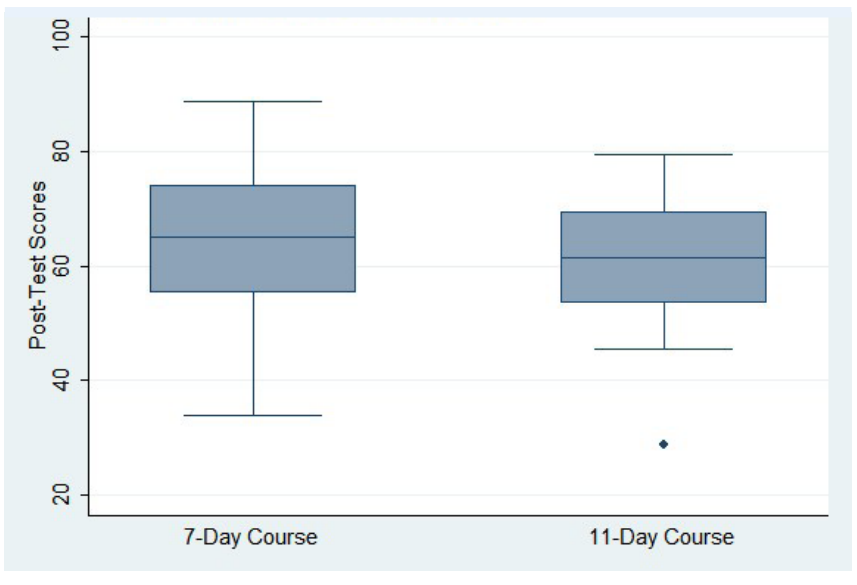

Figure 3. Post-test scores by course 
The pre-test scores in doctors were skewed as indicated by the median coinciding with the lower quartile value. Post-test score were less distributed than the pre-test scores. In nurses there was one under-performing outlier in the 11-Day course. In both groups midwives had the lowest pre-test scores and demonstrated the largest improvements (Figures 1 and 2). Improvements in test scores were greatest for midwives and doctors from the 7-Day course compared to the 11-Day course but only the score difference for midwives was statistically significant (Table 2).

\section{Direct observation Integrated Management of Childhood} Illness protocol adherence

The morbidity and severity of illnesses were compared in children observed by the 7-Day trainees and by the 11-Day trainees in Table 3. Morbidities of the observed children were described by both the number and category of classifications provided by the trainees. No children were classified with a severe illness during the fourth round. The average number of classifications provided by the health workers was similar in the 11-Day (2.1 classifications) compared to the 7-Day group (1.9 classifications). Although similar proportions of observed children were classified with fever, the 11-Day trainees more often classified the child as having malaria than children observed by the 7 -Day trainees (71.4\% versus $55.3 \%$, $P<0.01)$. In the 7 -Day group, fever was more commonly linked to other causes, such as otitis media, sore throat, or pneumonia. Classification of cough/URI was more frequent in 11-Day course group (58.0\%) than the 7-Day course group (45.6\%) but the difference was not statistically significant.

The use of antibiotics was similar in both groups and a high proportion of those classifications requiring antibiotics (pneumonia, dysentery, very severe fever, any complications of measles, mastoiditis, acute otitis media and strep throat) were given antibiotics in both course groups $(96.5 \%$ in 7-Day group; $93.3 \%$ in 11-Day group). Two children treated by the 11-Day course trainees and one child treated by the 7 -Day course trainees were not given antibiotics when the classification suggested that the use of antibiotics was correct. The mean number of prescribed medications was similar in the 11-Day (1.7 medications per patient) and in the 7-Day trainees (1.6 medications per patient). The mean number of prescribed drugs per classification was the same in both course groups (0.9 drugs per classification).

Both groups demonstrated appropriate use of anti-malarial medications when the classification was given (100.0\%). Both groups used zinc and ORS appropriately (95.5\% for the 7-Day course and $100.0 \%$ for the 11-Day course) although two children in the 7-Day course group did not receive ORS and zinc when the classification should have been treated with these drugs. In accordance with IMCI guidelines, neither group used anti-motility medications.

The two groups performed similarly in all four summary implementation scores for assessment, classification, treatment and counselling (Table 4). The average amount of time taken per interview was 2.7 minutes longer in the 7 -Day group than in the 11-Day group. Small differences in scores were evident when stratified by health worker type specifically for the counselling indicator in which the physicians in the 7-Day course out-performed those in the 11-Day course (93.3\% versus $80.4 \%$ ), while midwives and nurses in the 7-Day course performed the same as in the 11Day course (78.2\% versus 79.8\%). Neither of these differences was statistically significant. This remained true when the data was analysed by gender (data not shown).

Additional analyses of the summary scores included: 1) observations of health workers who had never taken an IMCI course; 2) observations in which health workers gave only three or fewer classifications; and 3) summary variables stratified by assessor. None of these analyses resulted in significantly different summary scores.

\section{Costs}

The costs of the trainers' time and the participants' accommodation and meals were 625.00 US dollars for each 7-Day course and 973.00 US dollars for each 11-Day course. An estimate of opportunity costs for each course was based on a mean of 14.40 children younger than five years of age that were received each day in primary care facilities. For each trainee in the 11-Day IMCI training course, roughly 158 children less than five years of age would not have received care or may have received less than adequate care during the absence of the usual health worker who provided care for children if another health worker were available. For the 7 -Day course the comparable number was 100 . Since, on average, each IMCI training course consists of 20 participants from 20 different health facilities, 2,016 children would have received less than adequate attention during one 7-Day course and 3,160 sick children potentially would not have been seen or treated during one 11-Day course.

\section{Discussion}

This evaluation demonstrated that health workers trained in the 7-Day course performed equally well as those trained in the 11-Day course in terms of mean knowledge scores immediately after training and in assessment, classification, treatment and counselling during assessment visits 32 to 34 weeks after training and three assessment visits by seasoned supervisors. This study contributes some evidence that was identified as lacking by Rowe et al. who concluded that

Table 2. Difference in pre- and post-test scores by health worker and course

\begin{tabular}{|c|c|c|c|c|c|}
\hline \multirow[b]{3}{*}{ Health worker type } & \multicolumn{2}{|c|}{ 7-Day course } & \multirow{2}{*}{\multicolumn{2}{|c|}{$\begin{array}{c}\text { 11-Day course } \\
\begin{array}{c}\text { Knowledge score post-test compared } \\
\text { to pre-test }\end{array}\end{array}$}} & \multirow{3}{*}{$\boldsymbol{P}$-value* } \\
\hline & \multicolumn{2}{|c|}{$\begin{array}{c}\text { Knowledge score post-test compared } \\
\text { to pre-test }\end{array}$} & & & \\
\hline & $\mathbf{N}$ & $\mathrm{N}(95 \% \mathrm{Cl})$ & $\mathbf{N}$ & $\mathrm{N}(95 \% \mathrm{Cl})$ & \\
\hline Medical doctor & 5 & $24.6(9.9,39.3)$ & 10 & $21.0(9.8,32.2)$ & 0.66 \\
\hline Midwives & 14 & $36.2(30.3,42.2)$ & 12 & $26.1(18.5,33.7)$ & 0.03 \\
\hline Nurse & 11 & $21.8(13.6,30.0)$ & 9 & $20.2(9.8,30.7)$ & 0.79 \\
\hline Total & 30 & $29.0(24.2,33.8)$ & 31 & $22.7(17.8,27.7)$ & 0.07 \\
\hline
\end{tabular}

${ }^{*}$ Analysis of Variance (ANOVA); statistical significance $=0.05$ 
Table 3. Monitoring Visits: classification and treatment of sick children by IMCl course

\begin{tabular}{|c|c|c|c|}
\hline \multirow[b]{2}{*}{ Classification } & \multicolumn{2}{|c|}{ Observations $^{\mathrm{a}}$} & \multirow[b]{2}{*}{$P$-value* } \\
\hline & $\begin{array}{c}\text { 7-Day course } \\
\text { N }(95 \% \mathrm{Cl})\end{array}$ & $\begin{array}{c}\text { 11-Day course } \\
\text { N (95\% Cl) }\end{array}$ & \\
\hline Number & 75 & 72 & \\
\hline \multirow[t]{2}{*}{ Mean No. classifications per child } & $1.9(1.6,2.1)$ & $2.1(1.8,2.3)$ & 0.22 \\
\hline & $\mathrm{N}(\%)$ & $\mathrm{N}(\%)$ & \\
\hline Severe classification $^{1}$ & 0 & 0 & - \\
\hline Pneumonia & $10(21.7)$ & $14(28.0)$ & 0.48 \\
\hline Acute otitis media (AOM) & $19(41.3)$ & $18(36.0)$ & 0.59 \\
\hline Pharyngitis & $2(4.3)$ & $2(4.0)$ & 0.93 \\
\hline Cough/URI & $21(45.6)$ & $29(58.0)$ & 0.11 \\
\hline Diarrhoea & $44(58.6)$ & $36(50.0)$ & 0.17 \\
\hline Diarrhoea + dehydration & $10(22.7)$ & $4(11.1)$ & 0.17 \\
\hline Dysentery & $4(9.1)$ & $3(8.3)$ & 0.91 \\
\hline Persistent diarrhoea & $3(6.8)$ & 0 & - \\
\hline Fever & $47(62.7)$ & $49(68.1)$ & 0.51 \\
\hline Measles (complicated or not) & 0 & 0 & \\
\hline Malaria & $26(55.3)$ & $35(71.4)$ & $<0.01$ \\
\hline Other illness & $21(44.6)$ & $13(26.5)$ & 0.09 \\
\hline Malnutrition (all) & $3(5.3)$ & $2(2.7)$ & 0.68 \\
\hline Very low weight & $3(100.0)$ & $2(100.0)$ & \\
\hline Severe malnutrition & 0 & 0 & - \\
\hline Anaemia (all) & 0 & $1(1.4)$ & 0.31 \\
\hline Anaemia & 0 & $1(100.0)$ & - \\
\hline Severe anaemia & 0 & 0 & - \\
\hline None & 0 & 0 & - \\
\hline \multirow[t]{2}{*}{ No. drugs per classification per child } & $0.9(0.7,1.0)$ & $0.9(0.8,1.0)$ & 0.92 \\
\hline & $\mathrm{N}(\%)$ & $N(\%)$ & \\
\hline Classification requiring antibiotics ${ }^{b}$ & $29(38.6)$ & $30(41.2)$ & 0.72 \\
\hline Given antibiotic & $28(96.5)$ & $28(93.3)$ & 0.51 \\
\hline No antibiotic given & $1(3.4)$ & $2(6.7)$ & 0.50 \\
\hline Classification of diarrhoea $^{c}$ & $44(58.7)$ & $36(50.0)$ & 0.29 \\
\hline Given ORS and zinc & $42(95.5)$ & $36(100.0)$ & 0.22 \\
\hline Not given ORS and zinc & $2(4.5)$ & 0 & - \\
\hline Given anti-motility drug & 0 & 0 & - \\
\hline Classification requiring ORS or antibiotic & $59(78.7)$ & $53(73.6)$ & 0.09 \\
\hline Given appropriate ORS or antibiotic & $58(98.3)$ & $52(98.1)$ & - \\
\hline Not giving appropriate ORS or antibiotic & $1(1.7)$ & $1(1.9)$ & - \\
\hline Classified as malaria $^{d}$ & $26(34.7)$ & $35(48.6)$ & 0.32 \\
\hline Given anti-malarial & $26(100.0)$ & $35(100.0)$ & - \\
\hline Not given anti-malarial & 0 & 0 & - \\
\hline Summary scores & $\%(95 \% \mathrm{Cl})$ & $\%(95 \% \mathrm{Cl})$ & \\
\hline Assessment score & $92.6(90.8,94.5)$ & $93.6(91.4,95.8)$ & 0.51 \\
\hline Classification score & $94.7(89.5,99.9)$ & $95.6(91.1,100.0)$ & 0.72 \\
\hline Treatment score & $94.7(89.8,99.5)$ & $97.2(94.5,99.9)$ & 0.39 \\
\hline Counselling score & $81.2(76.4,86.1)$ & $80.0(75.1,84.9)$ & 0.68 \\
\hline Duration score & $25.8(24.1,27.4)$ & $23.1(21.4,24.8)$ & 0.03 \\
\hline
\end{tabular}

$\mathrm{IMCl}=$ Integrated Management of Childhood IIIness; ORS= oral rehydration salts.

${ }^{1}$ Severe classification includes all cases that require immediate referral: very severe disease, severe pneumonia, diarrhea with severe dehydration, very severe febrile disease, severe complicated measles, severe malnutrition and mastoiditis. These classifications are included in the broad categories of ARI, diarrhea, fever, malnutrition but are not included in the specific categories under each of these headings.

aMore than one classification per child resulted in sums of proportions adding to more than $100 \%$

${ }^{\mathrm{b}}$ According to algorithms in IMCl chart booklet: pneumonia, dysentery, very severe fever, any complications of measles, mastoiditis, acute otitis media, strep throat

'Diarrhoea with or without dehydration, dysentery, persistent diarrhoea of any severity

${ }^{\mathrm{d}}$ Malaria or very severe febrile disease

${ }^{*}$ Chi-Square statistic; statistical significance $=0.05$ 
Table 4. Summary scores at fourth monitoring visit by health worker

\begin{tabular}{|c|c|c|c|c|c|c|c|c|c|c|c|c|}
\hline \multirow{2}{*}{$\begin{array}{c}\text { Summary Indicators } \\
\text { at } 4^{\text {th }} \text { Monitoring } \\
\text { Visit }\end{array}$} & \multicolumn{6}{|c|}{ MD } & \multicolumn{6}{|c|}{ MW or nurse } \\
\hline & $\mathbf{N}$ & 7 days & $95 \% \mathrm{Cl}$ & $\mathbf{N}$ & 11 days & $95 \% \mathrm{Cl}$ & $\mathbf{N}$ & 7 days & $95 \% \mathrm{Cl}$ & $\mathbf{N}$ & 11 days & $95 \% \mathrm{Cl}$ \\
\hline Assessment Score & 15 & $92.0 \%$ & $(86.6,97.4)$ & 21 & $93.4 \%$ & $(89.9,97.0)$ & 57 & $92.7 \%$ & $(90.8,94.8)$ & 50 & $93.7 \%$ & $(90.9,96.4)$ \\
\hline Classification Score & 15 & $93.3 \%$ & $(79.0,107.6)$ & 21 & $90.5 \%$ & $(76.7,104.1)$ & 60 & $95.0 \%$ & $(89.3,100.6)$ & 51 & $98.0 \%$ & $(94.1,101.9)$ \\
\hline Treatment Score & 15 & $96.6 \%$ & $(89.5,103.8)$ & 21 & $95.2 \%$ & $(88.4,102.1)$ & 60 & $94.2 \%$ & $(88.3,100.0)$ & 51 & $98.0 \%$ & $(95.3,100.7)$ \\
\hline $\begin{array}{l}\text { Counselling Advice } \\
\text { Score }\end{array}$ & 15 & $93.3 \%$ & $(88.2,98.4)$ & 21 & $80.4 \%$ & $(72.3,88.6)$ & 59 & $78.2 \%$ & $(72.4,83.9)$ & 50 & $79.8 \%$ & $(73.6,86.0)$ \\
\hline $\begin{array}{l}\text { Average duration of } \\
\text { observation }\end{array}$ & 15 & 22.3 & $(18.3,26.2)$ & 21 & 23.1 & $(20.5,25.7)$ & 60 & 26.7 & $(24.8,28.5)$ & 51 & 23.1 & $(20.9,25.3)$ \\
\hline
\end{tabular}

there were too few studies with first tier study designs to make any conclusion about effectiveness (13). However, in contrast to Rowe et al's conclusion in their systematic review, that the 11-Day IMCI course was slightly more effective than shorter IMCI training courses, our evaluation suggests that trainees perform similarly in both courses. In Rowe et al's review, however, the three studies that directly compared post-training performance between short and standard IMCI courses also concluded that there was no significance difference in post-training performance between groups $(8,16)$.

Our second finding - physicians trained in the 7-Day course did slightly better than physicians trained in the 11-Day course in providing three reasons to return to the health centre and in counselling about breastfeeding, increased liquid intake and the importance of follow-up - is remarkable because, poor counselling has been identified as typical of physicians (21). We highlight, however, that the statistical power in this study was insufficient to demonstrate a difference and that pursuing this line of inquiry requires a larger study.

The findings of this study indicate that nurses and midwives performed as well as physicians in assessment, classification and treatment, when trained in a 7-Day course as long as three assessment visits were conducted. This is important since the initial IMCI effort in Afghanistan, was focused almost exclusively on training physicians. Our study confirms the findings of Huicho et al. that "IMCI training is associated with much the same quality of child care across different health worker categories, irrespective of the duration and level of preservice training" (22). Their further conclusion that "Strategies for scaling up IMCI and other child-survival interventions might rely on health workers with shorter duration of pre-service training being deployed to underserved areas" was similar to the perspective of MoPH after 2008 to expand IMCI training to nurses and midwives in an attempt to increase availability of trained health workers in areas where nurses or midwives were the highest level of trained health worker. The fact that non-physicians performed less well in the counselling score is surprising in light of the literature and additional research would be necessary to elucidate this further (21). Previous studies in Afghanistan have also identified that female health workers performed better in counselling and providing quality care to children, findings not substantiated by this evaluation $(10,19)$.

In this evaluation, two physicians in the 7-Day group and three physicians in the 11-Day group had previously completed an 11-Day IMCI course, yet when these five doctors were excluded from outcome variable calculations, minimal differences in test scores and in summary variable scores were demonstrated. Although this number was small, the observation could stimulate the question of how many other health workers in Afghanistan retain knowledge and practice from previous 11-Day trainings. This finding is in line with that of the IMCI Working Group in 2007 which revealed low IMCI implementation amongst previously trained health workers (almost exclusively doctors) but it differs from larger cross-sectional studies that have demonstrated some benefit of previous IMCI training on quality of care $(9,10,17)$.

The 7-Day trainees classified fever as malaria less often than the 11 -Day trainees ( $47 \%$ versus $71 \%$ ), an observation that could have resulted from a difference in malaria incidence in the region during the two month period of the evaluation or due to the fact that the 7-Day course algorithm for fever was stratified into high incidence and low incidence areas. The low-incidence algorithm was set up to classify fever more commonly as non-malaria; whereas the high-incidence algorithm was designed to identify malaria more often. Validators were instructed to follow the algorithm provided in the course taken by the trainee. Concordance with the validator was high in both groups. Both groups used antimalarial medications appropriate for the given classification and never missed treating malaria when the classification was given. Were the 7-Day course to be adopted in Afghanistan, the importance of ensuring adequate emphasis of the concepts in the fever module must be stressed to avoid underclassification of malaria.

Several advantages of using the shortened course include: a) the ability to train more health workers in settings like Afghanistan where turnover of trained health workers is common; b) reduced cost of training per health worker in countries that rely on external funding for healthcare, (a 36\% cost savings was projected per course given in Afghanistan, findings substantiated in Zambia, Uganda and Kosovo) (13); and c) the possibility to re-allocate resources to provide supervisory visits, an activity that this evaluation and others suggest can maintain the long-term benefits of shorter IMCI training courses $(9,10,20)$. In addition, shortening the course responds directly to requests of NGO health workers who implemented IMCI in rural and remote settings where health workers have expressed difficulties in taking courses that last 11 days. Other potential complementary interventions could focus on retention of trained staff and promoting job satisfaction as well as developing ways to easily share the knowledge with other health workers such as the availability of easy-to-use job aids or electronic sources of information accessible by cell phone that are currently available in the 
Tanzania or other distance learning options (23-27).

Similar to other studies, this evaluation suggests that regular supervisory visits, during which detailed feedback is provided to the health worker, may improve the quality of care $(5,9)$. In this evaluation, $95 \%$ of patients were treated according to IMCI guidelines (PTIG), an outcome that compared favourably with the median PTIG of $66 \%$ in the 29 studies reviewed by Rowe et al. (13). The counselling score of $80 \%$ compared favourably with the counselling index of $48 \%$ identified in the 2008 Balanced Score Card for Afghanistan (28).

The opportunity costs of the 11-Day course compared to the 7 -Day course were substantial. Per course given, an estimate of 1,144 more children were likely to receive no care during the average attended 11-Day course compared to the average attended 7-Day course due to the absence of trainees. This "hidden cost of training" was 57\% more for the 11-Day IMCI training course, a finding substantiated elsewhere (8).

Several limitations have been identified in this evaluation. The initial design of the study included time series data collection at 6 weeks, 3 months and 6 months after training completion. Protracted trainings and difficulties in travelling to the facilities at the planned times for the first three assessment visits led to a very high variability in time elapsed between initial training and observation visits. Hence a fourth visit was added, 30 to 32 weeks after completion of the training and a minimum of one month following the previous supervisory visit. At that point, the observed health workers from both training courses had received three assessment visits during which their IMCI performance had been assessed, scored and corrected where necessary.

The sample size limited the study's power to detect differences in test scores and in some summary variables when data were stratified by health worker categories. Although our sample composition was typical of the staffing pattern of health workers that required training and was deemed to be adequate for evaluation by $\mathrm{MoPH}$ and WHO, it was not randomly selected and therefore, could not be considered statistically representative of all Afghan health workers that required training. Due to resource constraints, our attempt to match the cohorts met limited success. As a result, the 7-Day group had a higher composition of females and non-doctors than the 11-Day group, a selection bias that was expected to have resulted in lower knowledge and performance in the health worker trainees of the 7-Day group. This effect was not observed and both course groups performed similarly.

The use of the validators as a gold standard caused some known biases inherent in this method of validation. Firstly, observation caused health workers to change their usual habits, an effect that was demonstrated by an average consultation time of 25 minutes during this evaluation when in fact, Afghan health workers spend an average of 7 minutes per visit $(21,28)$. Thus, this evaluation did not clarify how health workers behaved in the absence of an observer. Secondly, although the assessors and validators were experts in using IMCI, their skills specific to validating and assessing may have changed over time, an effect that would have had minimal impact on the evaluation since it would have been similar for both trainee groups, and was minimized by comparing data from a fourth monitoring visit. Another potential bias was that all validators and assessors were male physicians, and a wide-spread notion in Afghanistan proposed that IMCI was not suitable for nurses and midwives, although the literature shows otherwise (22). The team may have been more critical when observing non-physicians but since the performance in all health workers was relatively high this effect was probably small.

Environmental bias was minimized by using the same instructors for both courses, conducting the courses in the same location at similar times, selecting trainees in similar geographic areas that had similar conditions for disease prevalence and logistical difficulties. Despite these efforts, some selection bias could have influenced our findings.

\section{Conclusion}

This evaluation demonstrated that both courses were associated with similar health worker performance at a fourth monitoring visit, 32 weeks after training. In light of this information, the potential financial savings, the reduction in opportunity costs, the possibility of training more health workers, and the ease with which health workers in remote settings might participate in shorter courses, the Afghan MoPH replaced the 11-Day IMCI course with the 7-Day IMCI course for both in-service and pre-service training in July 2011. Further research is necessary to define the optimal frequency of assessment visits subsequent to 32 weeks that would maximize IMCI implementation. Findings from this evaluation could be substantiated in a more in depth study that uses a larger sample size and multi-level analyses to control for the effects of health system factors and other confounders that have previously been associated with health worker performance and were not addressed in this evaluation. A larger sample size would be more likely to include children with severe classifications, a group that was absent in this evaluation.

In the absence of clear guidance on how and when to develop short IMCI courses that uphold quality, this article contributes to global knowledge, illustrating that evidencebased decisions can be made by complementing the existing literature with country-specific evaluations that allow adequate yet contextualized responses to local concerns regarding universal, standardized approaches.

\section{Acknowledgements}

The work that was the basis for this article was supported by funding from the U.S. Agency for International Development under the BASICS project, managed by Management Sciences for Health, contract number GHA-I-00-04-00002-00.

\begin{abstract}
Ethical issues
No ethical issues were identified. The Afghan Ministry of Public Health (MoPH) considered this evaluation part of quality improvements in routine care. The consent of healthcare workers to participate in this evaluation was implied when they registered for courses that included subsequent monitoring visits geared to reinforce knowledge from the course. Aggregate, de-identified data were presented.
\end{abstract}

\section{Competing interests}

The authors declare that they have no competing interests.

Authors' contributions

$\mathrm{MM}, \mathrm{PI}, \mathrm{HS}$ and SAA contributed to the design, pilot testing and modification of 
the 7-Day IMCl course. $\mathrm{MM}$ and $\mathrm{PI}$ contributed to the design of the evaluation. $\mathrm{PI}, \mathrm{HS}, \mathrm{WN}$ and SAA contributed to the implementation of the training and evaluation. PI and MM conducted the analysis of the data. All authors contributed to the interpretation of the findings and the writing of the manuscript.

\section{Authors' affiliations}

${ }^{1}$ School of Population and Public Health, University of British Columbia, Vancouver, BC, Canada. ${ }^{2}$ BASICS/Afghanistan and Centre for Health Services, Management Sciences for Health, Medford, MA, USA. ${ }^{3}$ BASICS/Afghanistan, Ministry of Public Health, Great Massoud Circle, Kabul, Afghanistan. ${ }^{4}$ Child and Adolescent Health Department, Ministry of Public Health, Kabul, Afghanistan.

\section{Additional files}

Additional file 1: Contains the Appendix 1.

Additional file 2: Contains the Appendix 2.

\section{References}

1. Salama P. Status of the health and nutrition sector in Afghanistan: progress and challenges 2001-2003. Kabul: UNICEF; 2003. Unpublished presentation by the Chief Health and Nutrition Officer of UNICEF.

2. The United Nations Children's Fund (UNICEF). Best Estimates of Social Indicators for Children in Afghanistan. New York City: UNICEF; 2005.

3. Gove S. Integrated management of childhood illness by outpatient health workers: technical basis and overview. The WHO Working Group on Guidelines for Integrated Management of the Sick Child. Bull World Health Organ 1997; 75 Suppl 1: 7-24.

4. Afghan Ministry of Public Health (MoPH). Basic Package of Health Services. Kabul: Afghan Ministry of Public Health; 2005.

5. Goga AE, Muhe LM. Global challenges with scale-up of the integrated management of childhood illness strategy: results of a multi-country survey. BMC Public Health 2011; 11: 503. doi: 10.1186/1471-2458-11-503

6. Goga AE, Muhe LM, Forsyth K, Chopra M, Aboubaker S, Martines $\mathrm{J}$, et al. Results of a multi-country exploratory survey of approaches and methods for $1 \mathrm{MCl}$ case management training. Health Res Policy Syst 2009; 7: 18. doi: 10.1186/1478-4505-718

7. Nguyen DT, Leung KK, Mclntyre L, Ghali WA, Sauve R. Does integrated management of childhood illness (IMCl) training improve the skills of health workers? A systematic review and meta-analysis. PLoS One 2013; 8: e66030. doi: 10.1371/journal. pone. 0066030

8. World Health Organization (WHO). Report of technical consultation on $\mathrm{IMCl}$ training approaches and pre-service IMCl: 19-23 November, 2007. Geneva: WHO; 2008.

9. Hansen PM, Peters DH, Niayesh $H$, Singh LP, Dwivedi $\mathrm{V}$, Burnham $\mathrm{G}$. Measuring and managing progress in the establishment of basic health services: the Afghanistan health sector balanced scorecard. Int J Health Plann Manage 2008; 23: 107-17. doi: 10.1002/hpm.931

10. Edward A, Kumar B, Niayesh H, Naeem AJ, Burnham G, Peters $\mathrm{DH}$. The association of health workforce capacity and quality of pediatric care in Afghanistan. Int J Qual Health Care 2012; 24: 578-86. doi: 10.1093/intqhc/mzs058

11. Chopra M, Patel S, Cloete K, Sanders D, Peterson S. Effect of an $\mathrm{IMCl}$ intervention on quality of care across four districts in Cape Town, South Africa. Arch Dis Child 2005; 90: 397-401. doi: 10.1136/adc.2004.059147

12. Bryce J, Victora CG, Habicht JP, Vaughan JP, Black RE. The multi-country evaluation of the integrated management of childhood illness strategy: lessons for the evaluation of public health interventions. Am J Public Health 2004; 94: 406-15.

13. Rowe AK, Rowe SY, Holloway KA, Ivanovska V, Muhe L, Lambrechts $\mathrm{T}$. Does shortening the training on Integrated
Management of Childhood Illness guidelines reduce its effectiveness? A systematic review. Health Policy Plan 2012; 27: 179-93. doi: 10.1093/heapol/czr033

14. Bishai D, Mirchandani G, Pariyo G, Burnham G, Black R. The cost of quality improvements due to integrated management of childhood illness (IMCI) in Uganda. Health Econ 2008; 17: 5-19. doi: 10.1002/hec.1231

15. Rowe AK, Osterholt DM, Kouame J, Piercefield E, Herman $\mathrm{KM}$, Onikpo F, et al. Trends in health worker performance after implementing the Integrated Management of Childhood Illness strategy in Benin. Trop Med Int Health 2012; 17: 438-46. doi: 10.1111/j.1365-3156.2012.02976.x

16. Tavrow P, Kekitiinwa Rukyalekere A, Maganda A, Ndeezi G, Sebina-Zziwa A, Knebel E. A comparison of computer-based and standard training in the Integrated Management of Childhood IIIness in Uganda. Bethesda, Maryland: United States Agency for International Development (USAID) through the Quality Assurance Project; 2002.

17. Afghan Ministry of Public Health (MoPH). Mid-Term Review of Facility-based Integrated Management of Childhood IIIness Implementation in Afghanistan. Kabul: MoPH Afghanistan/ USAID; 2008.

18. Afghan Ministry of Public Health (MoPH). Integrated Management of Childhood IIIness Situational Analysis. Kabul: BASICS/USAID; 2008.

19. Lind A, Edward A, Bonhoure P, Mustafa L, Hansen P, Burnham $\mathrm{G}$, et al. Quality of outpatient hospital care for children under 5 years in Afghanistan. Int J Qual Health Care 2011; 23: 108-16. doi: 10.1093/intqhe/mzq081

20. Edward A, Dwivedi V, Mustafa L, Hansen PM, Peters DH, Burnham $G$. Trends in the quality of health care for children aged less than 5 years in Afghanistan, 2004-2006. Bull World Health Organ 2009; 87: 940-9. doi: 10.2471/BLT.08.054858

21. Peters DH, Noor AA, Singh LP, Kakar FK, Hansen PM, Burnham G. A balanced scorecard for health services in Afghanistan. Bull World Health Organ 2007; 85: 146-51.

22. Huicho L, Scherpbier RW, Nkowane AM, Victora CG. How much does quality of child care vary between health workers with differing durations of training? An observational multicountry study. Lancet 2008; 372: 910-6. doi: 10.1016/S01406736(08)61401-4

23. Mitchell M, Hedt-Gauthier BL, Msellemu D, Nkaka M, Lesh N. Using electronic technology to improve clinical care - results from a before-after cluster trial to evaluate assessment and classification of sick children according to Integrated Management of Childhood Illness (IMCl) protocol in Tanzania. BMC Med Inform Decis Mak 2013; 13: 95. doi: 10.1186/14726947-13-95

24. Mitchell M, Getchell M, Nkaka M, Msellemu D, Van Esch J, Hedt-Gauthier B. Perceived improvement in integrated management of childhood illness implementation through use of mobile technology: qualitative evidence from a pilot study in Tanzania. J Health Commun 2012; 17 Suppl 1: 118-27. doi: 10.1080/10810730.2011.649105

25. World Health Organization (WHO). Integrated Management of Childhood Illness - Self-study modules. Geneva: WHO; 2014.

26. Lehmann U, Dieleman M, Martineau T. Staffing remote rural areas in middle- and low-income countries: a literature review of attraction and retention. BMC Health Serv Res 2008; 8: 19. doi: 10.1186/1472-6963-8-19

27. Quality Assurance Project. Evaluation of an $I \mathrm{MCl}$ Computerbased Training Course in Kenya. Bethesda, Maryland, USA: Published for the U.S. Agency for International Development (USAID) by the Quality Assurance Project; 2006.

28. Afghan Ministry of Public Health (MoPH). Afghanistan Health Sector Balanced Scorecard 2008. Kabul: MoPH; 2008. 